\title{
The Features of the Modeling the Nanofluid Flows
}

\author{
Valery Rudyak $^{1, \text { a) }}$ and Andrey Minakov ${ }^{1,2, \text { b) }}$ \\ ${ }^{1}$ Novosibirsk State University of Architecture and Civil Engineering, Russian Federation \\ ${ }^{2}$ Siberian Federal University, Krasnoyarsk, Russian Federation \\ a)valery.rudyak@mail.ru \\ b)aminakov@sfu-kras.ru
}

\begin{abstract}
The features of the nanofluid flows modeling are analyzed. In the first part the thermophysical properties (viscosity and thermal conductivity) of nanofluids are discussed in detailed. It was shown that the transport coefficients of nanofluids depend not only on the volume concentration of the particles but also on their size and material. The viscosity increases with decreasing the particle size while the thermal conductivity increases with increasing the particle size. The heat transfer of nanofluid in cylindrical channel and laminar-turbulent transition in some flows are considered. The heat transfer coefficient is determined by the flow mode (laminar or turbulent) of the nanofluid. However it was shown that adding nanoparticles to the coolant significantly influences the heat transfer coefficient. The laminar-turbulent transition begins in all cases earlier (at smaller Reynolds numbers) than for base fluid. In conclusion the possibility of the use of traditional similarity criteria are discussed.
\end{abstract}

Keywords. Nanofluids, viscosity, thermal conductivity, laminar-turbulent transition.

\section{INTRODUCTION}

Nanofluids are new two-phase system consisting of base liquid (water, ethylene glycol, etc.) and solid nanoparticles, usually metallic or oxide. The interest in nanofluids is associated with two main factors. On the one hand there are numerous existing or future applications of nanofluids (new systems for transportation and production of thermal energy; new coolants; biotechnology, new medicinal preparations and cosmetic products; drug delivery systems; different contamination detectors, water cleanup systems, and air cleaning units; new lubricants, lacquers, paints, etc.). On the other hand the nanofluids have unusual properties and their thermophysical characteristics are not described by the classical theories. Tens scientific teams around the world study the properties of nanofluids and their flows during last two decades. The obtained results proved extremely contradictory. Nevertheless, today it has been achieved a definite understanding of the physics of transport processes in nanofluids. Knowledge of the correct transport coefficients of nanofluids is absolutely necessary for adequate modeling of their flows. Therefore the present paper is devoted to an analysis of the thermophysical properties (viscosity, thermal conductivity and heat transfer coefficients) of nanofluids. The recent experimental and molecular dynamics data obtained by the authors are considered.

The second part of the paper deals with the study of two important flows. The heat transfer of nanofluid in tube and laminar turbulent transition in Poiseulle flow are considered. It was shown that the heat transfer coefficient is determined by the flow mode (laminar or turbulent) of the nanofluid. The use of the nanofluids as a coolant significantly affect on the magnitude of the heat transfer coefficient. The laminar-turbulent transition begins in all cases earlier (at smaller Reynolds numbers) than for carrier fluid.

In conclusion the possibility of the use of traditional similarity criteria are discussed. In particular, it is shown that the variation in the Prandtl number during modeling does not allow obtaining adequate information about the flow under study, since each point of the obtained dependences corresponds to different nanofluids simply. In this paper we used the experimental and molecular dynamics methods to study the thermophysical properties of nanofluids. The laminar-turbulent transition in nanofluid flow is studied numerically. 


\section{VISCOSITY AND THERMAL CONDUCTIVITY OF NANOFLUIDS}

Viscosity coefficient of the coarse dispersed fluids is described by the famous Einsten's formula $\eta=\eta_{0}(1+2.5 \phi)$, where $\eta_{0}$ is the viscosity coefficient of the based liquid and $\phi$ is the volume concentration of the particles. This formula is applicable only at very small particles concentration. There are very different theories and experimental correlations generalizing this formula. However in all cases the viscosity coefficient of dispersed fluid depends on concentration of the particles only. For a long time researchers of the viscosity of nanofluids also believed that it depends only on the concentration of nanoparticles. Therefore the experimental data were very different and nonuniversal. It was shown that in all cases the viscosity of nanofluids is much more than that of coarse dispersed liquid. The viscosity coefficient at small particles concentration is again described by the liner relation $\eta=\eta_{0}(1+a \phi)$ but the coefficient $a$ is varied from 4.3 to 22 (in Einstein's formula $a=2.5$ ).

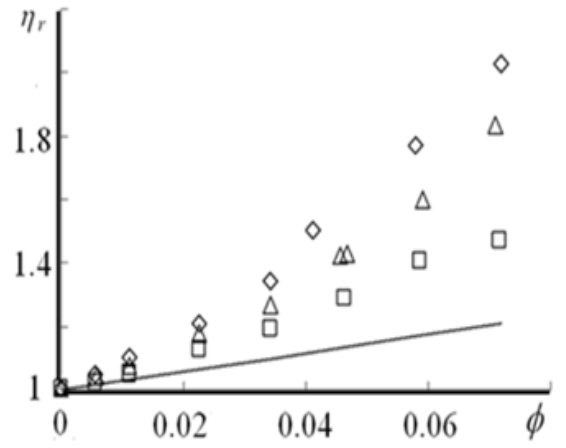

(a)

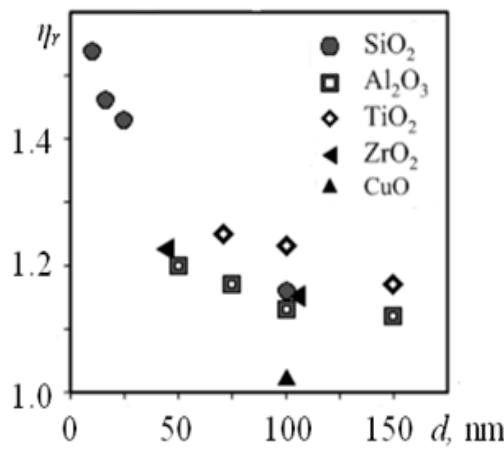

(b)

FIGURE 1. Relative viscosity $\eta_{r}=\eta / \eta_{0}$ of ethylene glycol based nanofluid with $\mathrm{SiO}_{2}$ nanoparticles versus the volume concentration (a) and size $d$ (b) of nanoparticles

More than ten years ago in our paper [1] it was firstly shown by molecular dynamics method that the effective viscosity coefficient for nanofluids depends not only on the concentration of particles but also on their size. Practically simultaneously this conclusion was confirmed experimentally. However there were very contradictory points of view about character of this dependence. In order to give an unambiguous answer, we measured the viscosity of more than fifty nanofluids. The measurements were performed using different rotational viscometers at a controlled temperature, and their accuracy was $1-2 \%$. The first data for nanofluids based on ethylene glycol with $\mathrm{SiO}_{2}$ nanoparticles are presented in Fig. 1a [2]. Here rhombuses correspond to particles with an average size of $18.1 \mathrm{~nm}$, triangles to 28.3 $\mathrm{nm}$, squares to $45.6 \mathrm{~nm}$, and the line corresponds to Einstein's value. The viscosity of all nanofluids considered is different and larger than Einstein's value.

The data shown in Fig. 1a clearly indicate that the nanofluid viscosity increases with decreasing particle size. Nevertheless, to remove any doubt, we measured the viscosities of more than fifty water-based nanofluids with different oxide particles. Some of these data are shown in Fig. 1b. The results of these measurements unambiguously suggest that the viscosity of nanofluids decreases with increasing nanoparticle size. This conclusion is confirmed by molecular dynamic modeling [3, 4].

The viscosity of nanofluids may depend not only on the size but also on the material of nanoparticles. This dependence is clearly seen in Fig. $1 \mathrm{~b}$ for nanofluids with $100 \mathrm{~nm}$ diameter particles. Previously, this dependence has been detected first using the molecular dynamics method [3] and then experimentally [5].

Most expectations have long been associated with various thermal applications of nanofluids. As late as the first experiments on measurement of their thermal conductivity showed excellent results: the incorporation of even small, of the order of a percent fraction or less, concentrations of solid metal nanoparticles enhanced the thermal conductivity of the base fluid by several percent or even tens of percent (see reviews [6-8]). This initiated a number of thermal conductivity measurements of the nanofluids, though the obtained data proved to be surprisingly controversial. It was found that the thermal conductivity of nanofluids is not described by the classical theories (Maxwell's theory and its generalizations). The first question that arises in the study of thermal conductivity of any dispersed fluid is formulated quite simply: how does the thermal conductivity depend on the particle concentration. The systematic measurements 
of thermal conductivity dependence on different parameters have been carried out in our recent paper [9]. More than fifty various nanofluids based on distilled water, ethylene glycol, and engine oil containing particles of $\mathrm{SiO}_{2}, \mathrm{Al}_{2} \mathrm{O}_{3}$, $\mathrm{TiO}_{2}, \mathrm{ZrO}_{2}, \mathrm{CuO}$, and diamond were studied. The nanoparticles volume concentration ranged from 0.25 to $8 \%$. The particle size ranged from 5 to $151 \mathrm{~nm}$. Thermal conductivity measurements were performed by non-stationary hot-wire method [10]. The error of fluid thermal conductivity coefficient does not exceed $3 \%$.

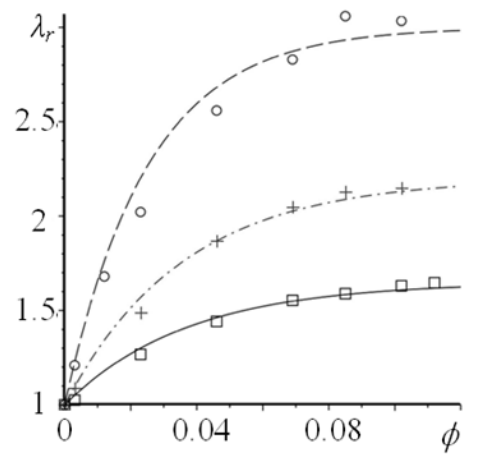

(a)

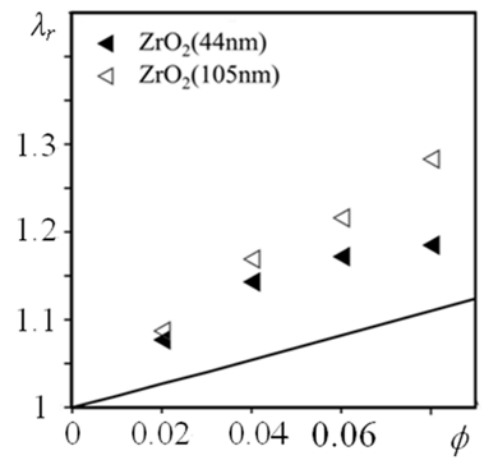

(b)

FIGURE 2. Relative thermal conductivity $\lambda_{r}=\lambda / \lambda_{0}\left(\lambda_{0}\right.$ is the thermal conductivity of the based fluid $)$ versus the particles volume concentration. Molecular dynamics [11] (a) and experimental (b) data [9].

Typical molecular dynamics data is presented in Fig. 2a. Here the molecular dynamics data obtained for nanoparticles with the same diameter $d=4 \sigma$ ( $\sigma$ is the effective size of the based fluid molecule) but for different masses $M / m: \square-M / m=80,+-M / m=100, \circ-M / m=150(M$ and $m$ are the mass of the nanoparticle and carrier fluid molecule respectively). A characteristic feature of the nanofluid thermal conductivity is slowdown of its enhancement with increasing of particle concentration. This dependence can be approximated by a simple formula $\lambda_{r}=1+b_{1} \varphi-b_{2} \varphi^{2}$. This behavior is observed experimentally. Experimental dependence of the thermal conductivity of two nanofluids is presented in Fig. 2b. Here the enhancement of nanofluid thermal conductivity over that of based fluid is greater (by 6-30\%) than the values defined by Maxwell's theory (solid line in Fig. 2b).

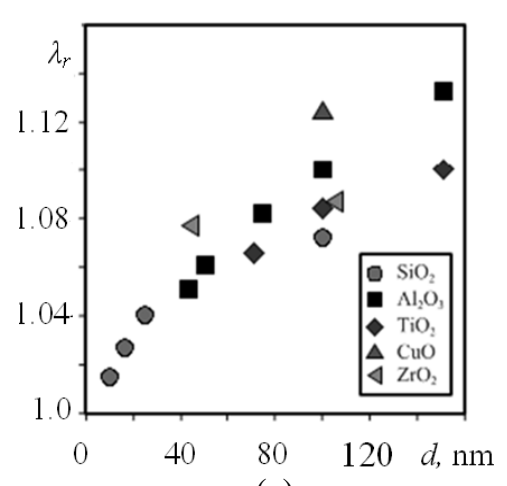

(a)

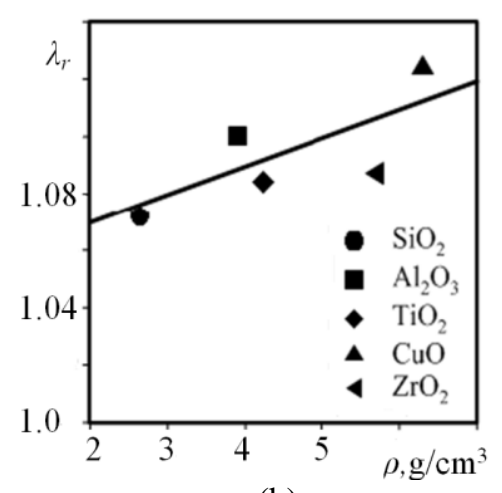

(b)

FIGURE 3. Relative thermal conductivity of nanofluids versus particle diameter (a) and particle material density for a particle volume concentration of $2 \%$ and a particle size of $100 \mathrm{~nm}$

The second important step was to determine the dependence of the thermal conductivity on the particle size. It is established that the thermal conductivity increases with increasing particle size (see Fig. 3a). How can the dependence of the thermal conductivity on the particle size be described? An analysis [12] of numerous experimental data has been shown that the relative thermal conductivity coefficient of a water based nanofluid with $\mathrm{Al}_{2} \mathrm{O}_{3}$ particles can be satisfactorily approximated by the formula: $k_{r}=1+A \sqrt{\varphi D}$, where $D=d / \sigma$ and constant $A$ should be depended on the nanoparticles material. The data presented in Fig. $3 \mathrm{a}$ also indicate that the thermal conductivity of nanofluids 
depends greatly on the particle material (see nanofluids with $100 \mathrm{~nm}$ diameter particles). All classical theories of the thermal conductivity of fluids with coarse dispersed particles assume that their thermal conductivity depends on the thermal conductivity of the particles. However, systematic measurements for five water based nanofluids with $\mathrm{ZrO}_{2}$, $\mathrm{TiO}_{2}, \mathrm{SiO}_{2}, \mathrm{CuO}$, and $\mathrm{Al}_{2} \mathrm{O}_{3}$ particles with the same volume concentration and size have shown no direct correlation between the thermal conductivity of nanofluids and the particle material [9]. Also there is no correlation between the thermal conductivity of nanofluids and their thermal diffusivity and specific heat of the particle material.

On the other hand, molecular dynamic simulations $[11,13,14]$ have shown that the thermal conductivity of nanofluids is greater the higher the density of the nanoparticles. Experiments confirm this dependence (see Fig. 3b), and this dependence is nearly linear. The constant $A$ in correlation for relative thermal conductivity given above indeed depends on the density of the particle material. Accordingly, this correlation becomes: $\lambda_{r}=1+(0.0193+0.00383 \tilde{\rho}) \sqrt{\varphi D}$. Here $\tilde{\rho}=\rho_{p} / \rho_{0}, \rho_{p}, \rho_{0}$ are the density of the nanoparticle and base fluid materials, respectively. This formula is good agreed with the experimental data (the error is less than $3 \%$ for the water based nanofluids).

The base liquid also significantly influences the effective thermal conductivity of the nanofluid. We have confirmed that the lower the thermal conductivity of the base fluid, the higher the enchancement of the thermal conductivity of the nanofluid. This, in particular, means that the supplement of the nanoparticles into the fluid will be most effective for the base fluid with low thermal conductivity.

\section{NANOFLUID HEAT TRANSFER}

The heat transfer coefficient of a nanofluid in a cylindrical channel under constant heat flux density at the walls was measured experimentally. The experimental setup was described in detail in $[15,16]$. It was a closed loop with a circulating coolant. The working fluid was circulated by a pump through the heated measuring section. The heated section was a stainless steel tube $6 \mathrm{~mm}$ in diameter and $1 \mathrm{~m}$ long. The tube was heated by supplying an electric current directly to its wall. Local temperature of the tube was measured with six copper-constantan thermocouples attached to its walls. In addition, the temperature at the inlet and outlet of the heated section was measured using thermocouples. Pressure gradient was measured with a differential pressure gauge. The measurement error was $0.1 \%$ for the pressure gradient and about $1 \%$ for temperature. Heat transfer was studied for several water-based nanofluids with $\mathrm{CuO}$ (55 $\mathrm{nm}), \mathrm{TiO}_{2}(100 \mathrm{~nm}), \mathrm{ZrO}_{2}(105 \mathrm{~nm}), \mathrm{SiO}_{2}(10,16,25,100 \mathrm{~nm})$, and $\mathrm{Al}_{2} \mathrm{O}_{3}(10,25,100 \mathrm{~nm})$ particles. The particle concentration was varied from 0 to $2 \%$.

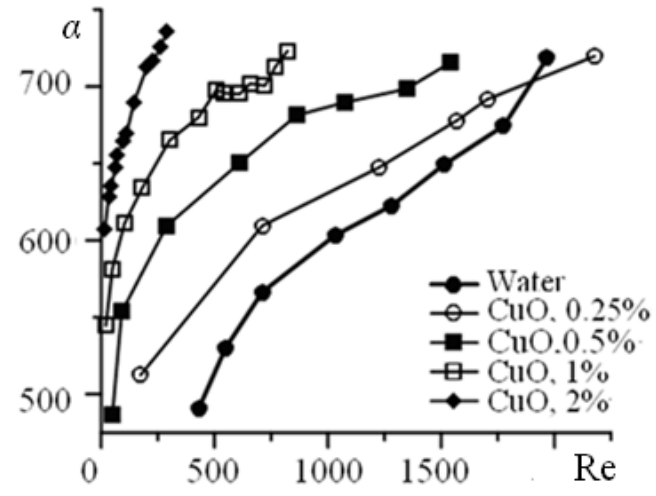

(a)

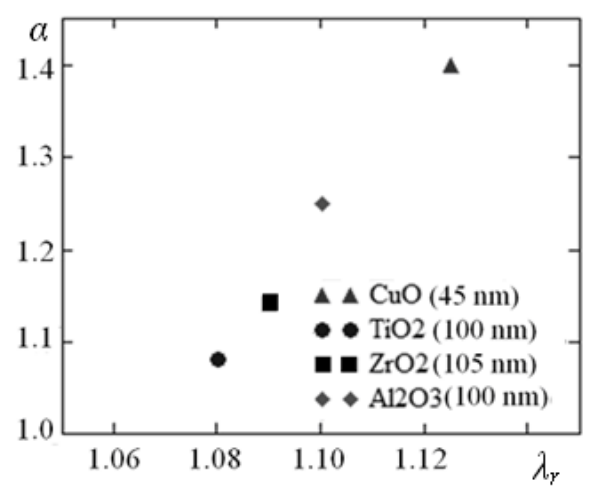

(b)

FIGURE 4. Average heat transfer coefficient versus Reynolds number (a) and relative thermal conductivity of nanofluids for different nanoparticle materials at constant Reynolds number (b)

It has been shown that using the nanofluids as a coolant significantly affect on the magnitude of the heat transfer coefficient. In laminar flow the heat transfer coefficient of nanofluids in all cases is much more than that of based fluid. The relative heat transfer coefficient $\alpha=\alpha_{n} / \alpha_{0}\left(\alpha_{n}\right.$ and $\alpha_{0}$ are the heat transfer coefficients of the nanofluid and the base liquid respectively) of nanofluids with $\mathrm{CuO}$ nanoparticles (see Fig. $4 \mathrm{a}$ ) increases rapidly with increasing their concentration: for the one-percent nanofluid, it is more than $40 \%$ higher than that for water at practically all 
Reynolds numbers (Re), and at a particle concentration equal to $2 \%$, it is more than twice that for water. Naturally, with a decrease in the concentration of nanoparticles, this effect decreases monotonically. In laminar flow, the heattransfer coefficient increases almost linearly with increasing thermal conductivity of nanofluid. This is illustrated in Fig. 4b, which shows the dependence of the relative heat transfer coefficient on the relative thermal conductivity.

The effect of using nanofluids in turbulent mode is much more complicated. In this case the heat transfer coefficient depends not only on the thermal conductivity of nanofluid but also on its viscosity. However it was shown that adding nanoparticles to the coolant significantly influences the heat transfer coefficient. It is shown that with increasing nanoparticles concentration, the local and average heat transfer coefficients at a fixed Reynolds number increase. Decrease in heat transfer coefficient with increasing particles concentration may take place at a fixed flow rate. It is shown that, the heat transfer coefficient of the nanofluid in turbulent regime increases with increasing nanoparticles size at a fixed flow rate, while has a certain maximum at a fixed Reynolds number. It is found that the inlet temperature is another factor having a significant effect on turbulent heat transfer performance of nanofluids.

\section{LAMINAR-TURBULENT TRANSITION}

Practically all applications of nanofluids are connected with their flows along the channels. It is clear that the flow regime will be determined the performance of the corresponding tools (see previous section). However the laminarturbulent transition of the nanofluid flows has not practically studied. Investigation of the influence of nanoparticles on stability of cylindrical Poiseuille flow is the aim of this section. We consider the hydrodinamical stability of this flow with respect to small disturbances. We will analyze the stability of the flow of ethylene glycol based nanofluid with $\mathrm{SiO}_{2}$ particles. In this case the fluid density $\rho$ depends on volume concentration of nanoparticles: $\rho=(1-\phi) \rho_{0}+\phi \rho_{p}$, here $\rho_{p}$ and $\rho_{0}$ are the density of the nanoparticle material and based fluid, respectively. The fluid is Newtonian one and the particles distribution is supposed homogeneous. The viscosity coefficient of the nanofluids considered is determined by the experimental correlation [2] $\mu^{\prime}=1+\left(2.5+13.427 e^{-0.013 d / \sigma}\right) \varphi+\left(6.2+514.657 e^{-0.013 d / \sigma}\right) \varphi^{2}$.

Let us consider the stationary flow between two concentric cylinders induced by the pressure gradient along the channel axis $(z)$. This flow has the following velocity profile [17]

$$
W_{0}(r)=-\frac{1}{4 \mu} \frac{\partial P}{\partial z}\left(R_{i}^{2}-r^{2}+\frac{R_{o}^{2}-R_{i}^{2}}{\ln \left(R_{o} / R_{i}\right)} \ln \left(r / R_{i}\right)\right),
$$

here $R_{i}, R_{o}$ are the radius of the inner and outer cylinders, respectively.

In the present paper, the stability of the cylindrical Poiseuille flow with respect to axisymmetric small disturbances has been studied. In this case the azimuthal modes of velocity perturbations are absent, $u_{\theta}=0$, and radial $u_{r}$ and axial $u_{z}$ velocity disturbances are expressed through the stream function $\Psi$ as follows: $u_{r}=-(\partial \Psi / \partial z)$, $u_{z}=\partial \Psi / \partial r+\Psi / r$. Solution for the stream function is sought in the form of normal modes $\Psi=\psi \exp [i \alpha(z-C t)]$. In this case for the amplitude of the disturbances the known Orr-Zommerfeld equation is obtained

$$
\left[L-\alpha^{2}+i \alpha \operatorname{Re}\left(C-W_{0}\right)\right]\left(L-\alpha^{2}\right) \psi+i \alpha \operatorname{Re}\left(W_{0}^{\prime \prime}-W_{0}^{\prime} / r\right) \psi=0, \quad L \equiv \frac{d^{2}}{d r^{2}}+\frac{1}{r} \frac{d}{d r}-\frac{1}{r^{2}},
$$

with corresponding boundary conditions $\left(\theta=R_{i} / R_{o}\right)$

$$
\psi\left(\frac{2 \theta}{1-\theta}\right)=\psi\left(\frac{2}{1-\theta}\right)=\psi^{\prime}\left(\frac{2 \theta}{1-\theta}\right)=\psi^{\prime}\left(\frac{2}{1-\theta}\right)=0 .
$$

Here $\operatorname{Re}=\rho \operatorname{Re}_{0} / \eta$ and $\operatorname{Re}_{0}$ are the Reynolds number of nanofluid and based fluid, respectively.

The solution of the stability problem consists in determining the dependence of the wave number $\alpha$ on the Reynolds number $\operatorname{Re}$ when $\operatorname{Im}(C)=0$. The problem was solved by the Galerkin method $[18,19]$. Neutral stability curves founded for cylindrical Poiseuille flow of nanofluid shown on Fig. 5a demonstrate significant destabilization of the nanofluids. Curve 1 in Fig. 5a is obtained for nanoparticles volume concentration $\phi=0.05$, curve $2-\phi=0.04$, curve 3 $\phi=0.03$, curve $4-\phi=0.02$, curve $5-\phi=0.01$, curve $6-\phi=0$. Destabilization effect grows monotonically with increasing of nanoparticles concentration. The critical Reynolds number increases almost threefold at a particle concentration of 5\%. Destabilization effect grows monotonically also with decreasing nanoparticles size. 


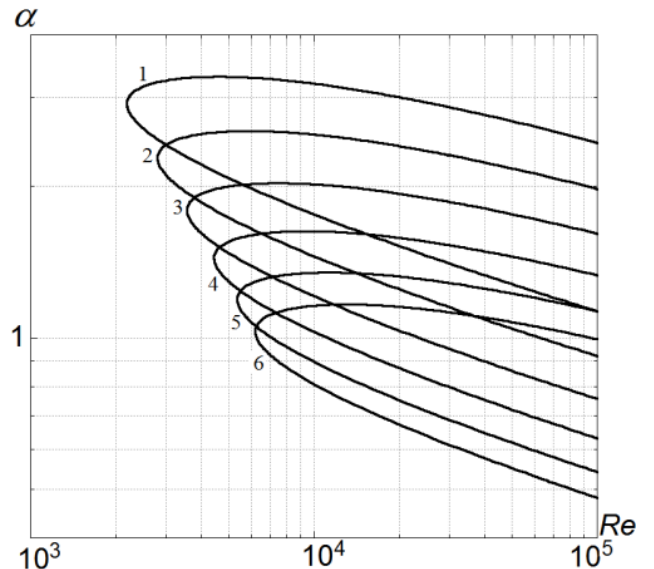

(a)

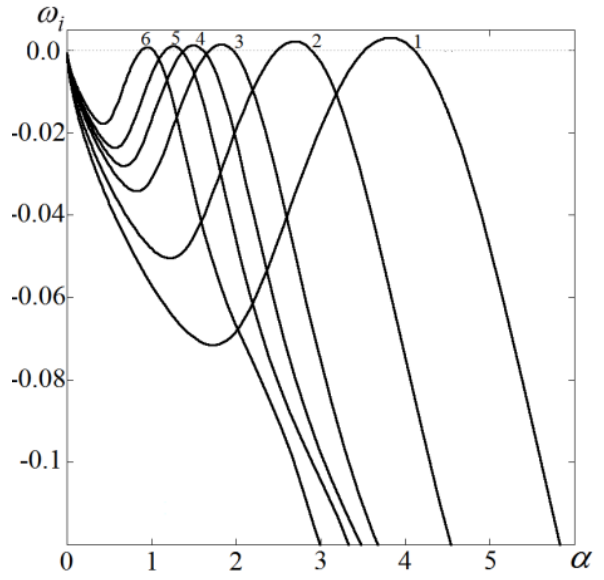

(b)

FIGURE 5. Neutral stability curves for nanofluids, $\theta=0.5 d=27.9 \mathrm{~nm}$ (a). Dependence of the growth increment of the unstable disturbance on wave number at different nanoparticles size (b)

The most important characteristic of flow stability is the value of the growth increment of the unstable disturbances. Dependence of the growth increment of the unstable disturbances on wave number is presented in Fig. $5 \mathrm{~b}$. Here the curve 1 corresponds to the particle size $17.6 \mathrm{~nm}, 2-27.9 \mathrm{~nm}, 3-45 \mathrm{~nm}, 4-60 \mathrm{~nm}, 5-90 \mathrm{~nm}, 6$ - to based fluid. There is the similarity between the curves $\omega_{i}(\alpha)$ in flows with different size of nanoparticles. The effect of nanoparticles is reduced to the extension of the dependence of function $\omega_{i}(\alpha)$ along the axis of wave numbers.

Thus the nanofluid flow is less stable than the flow of based fluid or in other words the critical Reynolds numbers of the laminar-turbulent transition for nanofluid flow are less than for based fluid. This conclusion is universal and it have been confirmed experimentally [20].

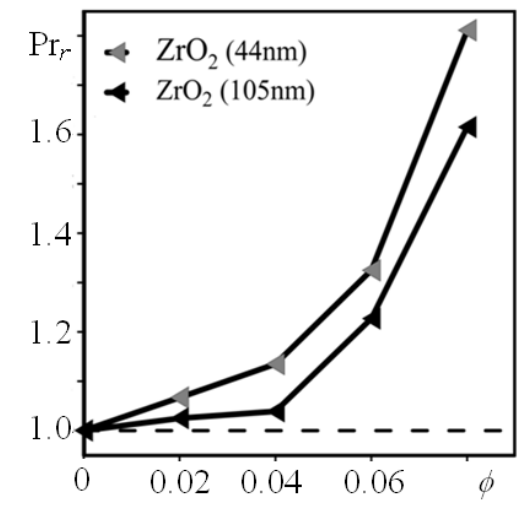

(a)

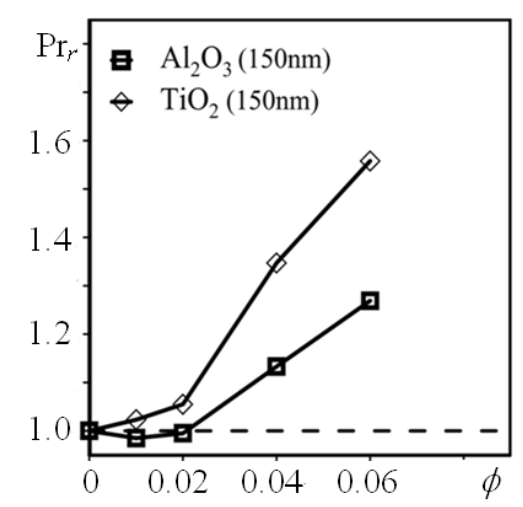

(b)

FIGURE 6. Concentration dependences of relative Prandtl number $\operatorname{Pr}_{r}=\operatorname{Pr} / \operatorname{Pr}_{0}$ of water based nanofluids with $\mathrm{ZrO}_{2}$ particles of different sizes (a) and different particles $\left(\mathrm{Al}_{2} \mathrm{O}_{3}, . \mathrm{TiO}_{2}\right)$ of the same size (b)

In practice, all applications and laboratory investigations of nanofluids deal with their flows. Description of the nanofluid flows, as well as usual fluids, typically employs well-known similarity criteria, such as Reynolds, Prandtl, Nusselt numbers and so on. The unusual thermophysical properties of nanofluids do not always permit the use of standard similarity parameters. For example, the Prandtl number $\operatorname{Pr}=C_{p} \eta / \lambda$ is a complex function of particle concentration, size, and material. Simple variation of this parameter in modeling nanofluid flows may be useless because the simulated flow property at each point will just correspond to different nanofluids. This is illustrated in Fig. 6, which shows the dependence of the relative Prandtl number $\operatorname{Pr}=\operatorname{Pr} / \operatorname{Pr}_{0}\left(\operatorname{Pr}_{0}\right.$ is the Prandtl number of the based fluid) on the nanoparticle concentration for two nanofluids with $\mathrm{ZrO}_{2}, \mathrm{Al}_{2} \mathrm{O}_{3}$ and $\mathrm{TiO}_{2}$ particles [21]. 


\section{CONCLUSION}

Thus, nanofluids are not conventional suspensions. Their thermophysical properties are not described by the classical theories. Today we can say with confidence that the viscosity of nanofluids is significantly higher than the viscosity of conventional coarse dispersed fluids. This conclusion is confirmed by recent experiments and molecular dynamics simulation. In contrast to the viscosity of coarse dispersed fluid, the viscosity of nanofluids depends not only on the particle concentration but also on their size and material.

The thermal conductivity of nanofluid also depends on size and material of nanoparticles. As a rule the thermal conductivity far exceed the value given by Maxwell's formula. It increases with increasing particle concentration, reaches a certain maximum, and then varies only slightly. In contrast to classical theories, the thermal conductivity of nanofluids depends on the nanoparticle size and increases with its increase. The effect of the thermal conductivity enhancement is determined not only by the nanoparticle size, but also by the density of the nanoparticle material. In this regard, we note that the mass concentration of nanoparticles in nanofluids increases dramatically with increasing density of the particle material. Thus, the nanofluid thermal conductivity enhancement over the value for the based fluid is greater, the higher the mass fraction of nanoparticles.

Strictly speaking the term nanofluids includes the media of different nature. We have to differentiate the nanosuspensions (carrier liquid+nanoparticles) and gas nano-suspensions (carrier gas+nanoparticles). It is evidently that to model these different classes of the dispersed fluids we need to use the different methods. Detailed discussion of these methods can be found in our monograph [22]. We confine ourselves here to only two remarks. It is necessary to use the kinetic theory to model the flows of the gas nano-suspensions if the carrier gas is rarefied. Practically in all other cases we may apply the hydrodynamical approach. As a rule the flows of the nanofluids are described by the one-fluid hydrodynamic model. However considering the dynamics of the non-isothermal flow we need in certain cases to use the two-fluid model.

A description of the microflows of nanofluids requires caution. There are the situations where the size of dispersed nanoparticles may not be small as compared with the characteristic size of the microchannel. In these cases we have to use the mixed kinetic-hydrodynamic description of the nanofluid [23]. In such approach the carrier fluid is described by the hydrodynamic but the pseudo-gas of nanoparticles is described by the kinetic equation.

Finally, it is revealed that in some cases, nanofluids are characterized by non-Newtonian rheological behavior. The emergence of non-Newtonian properties is caused by several factors, such as the concentration of nanoparticles, their size and material, as well as properties of the base fluid. The change in rheological behavior from Newtonian to nonNewtonian pattern occurs with increasing nanoparticle concentration. All investigated non-Newtonian nanofluids turned out to be either pseudoplastic or viscoplastic. Particle size is another important parameter determining the rheological behavior of nanofluids. It is revealed that the transition from Newtonian behavior to non-Newtonian one in some cases occurs at a given volume concentration with decreasing size of nanoparticles.

\section{ACKNOWLEDGMENTS}

The study has been conducted at partial financial support of the Russian Foundation for Basic Research (grants No. 17-01-00040 and No. 17-58-45032) and Russian Science Foundation (project No. 17-79-20218).

\section{REFERENCES}

1. V.Ya. Rudyak, A.A. Belkin, E.A. Tomilina and V.V. Egorov, preprint NSUACE 1(18), (2006).

2. V.Ya. Rudyak, S.V. Dimov, V.V. Kuznetsov and S.P. Bardakhanov, Doklady Physics 58(5), 173-176 (2013).

3. V.Ya. Rudyak and S.L. Krasnolutskii, Phys. Letters A 378, 1845-1849 (2014).

4. V.Ya. Rudyak and S.L. Krasnolutskii, Tech. Physics 60(6), 798-804 (2015).

5. V.Ya. Rudyak, A.V. Minakov, M.S. Smetanina and M.I. Pryazhnikov, Doklady Physics, 61(3), 152-154 (2016).

6. Yu. France, W. Routbort and D.M. Choi, Heat Transfer Eng. 29(5), 432-460 (2008).

7. C. Kleinstreuer and Y. Feng, Nanoscale Research Letters 6 229-242 (2011).

8. P. Keblinski, R. Prasher and J. Eapen, J. Nanoparticles Research 10 1089-1097 (2008). 
9. M.I. Pryazhnikov, A.V. Minakov, V.Ya. Rudyak and D.V. Guzei Int. J. Heat and Mass Transfer 104(1), 12751282 (2017).

10. A.V. Minakov, V.Ya. Rudyak, D.V. Guzei, M.I. Pryagnokov and A.S. Lobasov, J. Eng. Physics and Thermophysics 88(1), 149-162 (2015).

11. V.Ya. Rudyak and A.A. Belkin, Nanosystems: Physics, Chemistry, Mathematics, 1, 156-177 (2010).

12. D. Ceotto and V.Ya. Rudyak, Colloid J. 78(4), 509-514 (2016).

13. V.Y. Rudyak, A.A. Belkin and E.A. Tomilina, Tech. Phys. Letters 36(14), 49-54 (2010).

14. V.Ya. Rudyak and S.L. Krasnolutskii, Tech. Physics 62(10), 1456-1465 (2017).

15. A.V. Minakov, V.Ya. Rudyak, D.V. Guzei and A.S. Lobasov, High Temperature 53(2), 246-253 (2015).

16. A.V. Minakov, A.S. Lobasov, D.V. Guzei, M.I. Pryazhnikov and V.Ya. Rudyak, Applied Thermal Engineering 88, 140-148 (2015).

17. L.G. Loitsyanskii, Mechanics of Liquids and Gases (Pergamon Press, Oxford, 1966).

18. V. Rudyak, E. Isakov and E. Bord, J. Aerosol Sci. 28(1), 53-66 (1997).

19. V.Y. Rudyak, E.B. Isakov and E.G. Bord, Thermophysics and Aeromechanics 3(1), 51-56 (1996).

20. V.Y. Rudyak, A.V. Minakov, D.V. Guzei, K.N. Meshkov, V.A. Zhigarev and M.I. Pryazhnikov, Thermophysics and Aeromechanics 23(5), 773776 (2016).

21. V.Y. Rudyak, Minakov A.V. and M.I. Pryazhnikov, Tech. Phys. Letters 43(1), 23-26 (2017).

22. V.Y. Rudyak and A.V. Minakov, Modern Problems of Mmicro- and Nanofluidics. (Science, Novosibirsk, 2016).

23. V.Y. Rudyak Sov. Tech. Phys. Letters 25(22), 42-45 (1999). 